\title{
Healthcare ICT for Temporary Housing Community in Disaster-Stricken Area
}

Masahiro KURODA $^{\dagger a)}$, Nonmember

\begin{abstract}
SUMMARY Various information and communication technology (ICT) applications have been introduced for use in disaster-stricken areas. Of these, those for healthcare purposes proved useful when used for continually monitoring health conditions and easily using medical and healthcare devices, such as sphygmomanometers, which are familiar to the public. Devices that were easy to understand and use were more acceptable in the first post-disaster stage in a temporary housing community as victims were experiencing mental and physical trauma. After gaining the trust of medi$\mathrm{cal} /$ healthcare staff caring for the victims, we could move to the next stage with their support. ICT technologies are tools and are basically invisible to victims; a notion we have recognized anew. This paper introduces our activities in the area of healthcare monitoring services. The UMe-1 system and subset health check kiosk version 0 are introduced. The kiosk is simple and important for life support advisers in support centers as a tool for checking the health condition of residents. Not all those living in the target temporary housing community are currently using the kiosk due to its location within the widespread premises, but the director of a support center recognizes the utility of the kiosk and expects that a community-based kiosk and user-friendly BAN device could be used at home for mimamori (the Japanese concept of informally monitoring healthcare) because residents living far from support centers have difficulty regularly visiting the center, especially in the winter. We introduced a television-based, in-home health check kiosk and are currently working on its development. There are gaps between actually deployable technologies and research results, but it is also important to continue to address research issues concerning reducing impact through the user interface and introducing less stress to the everyday lives of disaster victims.
\end{abstract}

key words: healthcare, ICT, disaster, BAN, kiosk

\section{Introduction}

The Great East Japan Earthquake that struck off the Pacific coast of Japan's Tohoku region on March 11, 2011, was the largest in the country's recorded history and triggered a massive tsunami that killed thousands. After the disaster, many researchers visited the stricken area for using research-related applications, and a few of these were proven helpful in the area of healthcare services and benefitted from cooperative and continuous activity with local residents.

There are two stages in aiding people following largescale disasters. The first recovery stage, just after the event, is for providing rescue and medical treatment, with remuneration provided as per the Basic Act on Disaster Control Measures [1]. The second stage positioned from phase 3 and beyond, is for attempting to restore everyday life for

Manuscript received January 20, 2012.

Manuscript revised April 21, 2012.

The author is with National Institute of Information and Communications Technology, Koganei-shi, 184-8795 Japan.

a)E-mail: marsh@ @ict.go.jp

DOI: 10.1587/transcom.E95.B.3062

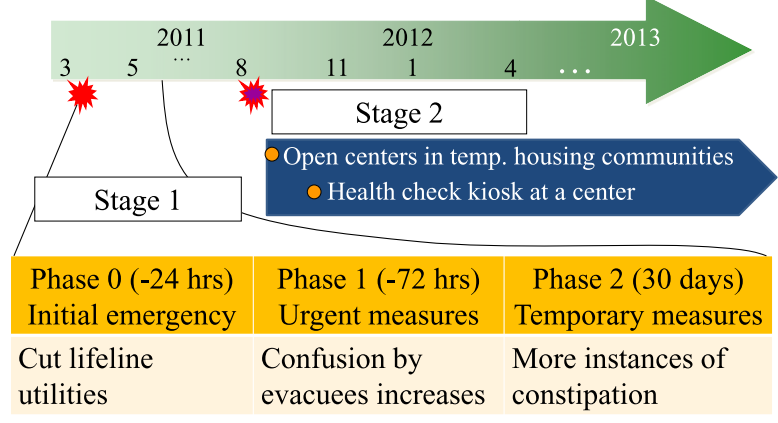

Fig. 1 Disaster recovery phases and stages.

evacuees after the emergency phases $0-2$. The Act does not financially support these and this makes it difficult for those outside of the region to work there (Fig. 1).

Various applications of information and communication technology (ICT) not only work to restore communications but also rebuild them for better services in the future. Body area networks (BANs) are useful in the second stage after a support center is constructed for collecting healthcare and medical data, such as those on physical and mental exams, under the management of life support advisers (LSAs) in a social welfare service organization that establishes a contract with local governments or offices.

This paper introduces our activity in the second recovery stage in the disaster-stricken area, as well as the physical environment. We then explain an experimental system that serves as the basis for the health check kiosk version 0 in support centers and a foreseen healthcare-related ICT system that includes the kiosks and a user-friendly BAN. The paper concludes by discussing future directions of this activity.

\section{Disaster Recovery Phases and Activities}

Disaster recovery is said to consist of four phases: the initial emergency phase, urgent measures, temporary measures, and restoration and rebuilding, though these phases and their lengths vary according to the nature and severity of the disaster.

We began work on our ICT-BAN deployment from August 2011, as per the schedule shown in Fig. 1, when a support center opened at the site of a temporary housing community. BANs are useful for LSAs to check health conditions and chronic illnesses. They are installed in temporary 
housing and have a network for automatically collecting all health-related data at the support center. It is difficult to install a BAN into each home as well as in the support center because of physical space limitations from surpluses of relief goods, and unfamiliar health checkups in the home add to the workload for LSAs. The BAN system could be accepted as a complementary tool for automatically monitoring a large number of people while staffed with a small number of LSAs.

We first demonstrated a health check kiosk based on the UMe-1 system, which is described later, at the Kamaishi medical association so that LSAs and city workers could gain a better understanding. The simple and actual, practical demonstration of the system, rather than a Powerpoint presentation, impressed those attending the meeting and both parties agreed to install a kiosk consisting of a sphygmomanometer and a data management PC with short-range wireless communication within a support center of a temporary housing community in Kamaishi city. We then installed the system at another support center in Otsuchi town based on the evaluation at the first support center.

\section{Temporary Housing Environment}

Following the tsunami, temporary housing was located in inconvenient areas because good locations were occupied by residential housing that had been destroyed. Tsunamisafe areas are typically located in between mountains, where cell phones are out-of-range, and temporary housing is also dotted in locations at the foot of mountains because of the limited space available in Iwate prefecture (Figs. 2, 3).

Some housing communities have limited communication facilities because housing was constructed in areas where no residents were living before the tsunami. Local governments provide a television set for each temporary home to provide broadcast information either via terrestrial broadcasting or cable television network. Other means of

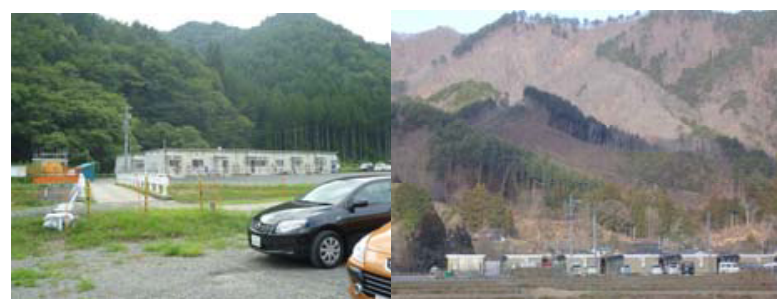

Fig. 2 Housing community in summer and winter.

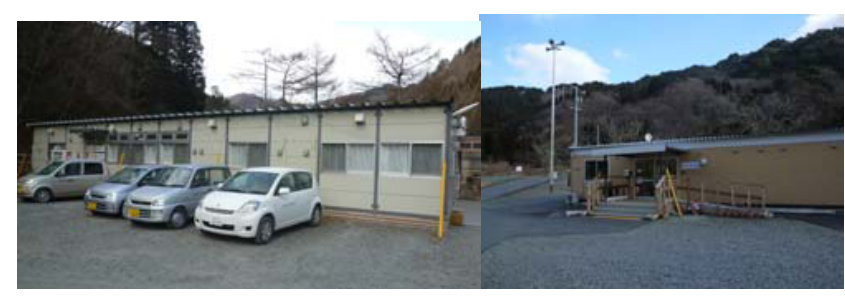

Fig. 3 Support centers in Kamaishi and Otsuchi. communication may not be available, for instance:

1) Cell phones may be out-of-range

2) FTTH/ADSL services may not be available

In addition to the communication environment, the following should be considered in deploying ICT devices.

3) Power voltage may not be stable

4) Very low winter temperatures and batteries may not be charged

5) Limited space is available in support centers and individual houses

6) People are experiencing mental strain

7) Some of the working personnel are also victims

8) Support center personnel and city workers are busy

These conditions need to be taken into account when introducing ICT technologies for ordinary people.

\section{Medical Examinations and Healthcare}

After the disaster, more than 13,000 temporary housing units were built in Iwate prefecture. ICT-BAN technologies are important for providing automatic care for people since they are under mental strain. The Japanese term mimamori is used to describe the process of taking care of people casually and indirectly, inquiring about health conditions in a friendly way, rather than directly monitoring, watching, or supervising. It is suitable for LSAs to deploy BANs for individuals with whom they have established relationships of trust through mimamori, since this provides them with measured and objective data, but it is difficult to attain consensus among the medical association, city office, and social welfare service organization managing the temporary housing community.

We designed an experimental self-health checkup system called UMe-1, which is targeted at mobile mass medical examination. Healthcare ICT systems deployable in disaster-stricken areas have functionality and an interface similar to that of the UMe-1. We first look briefly at the UMe-1 system and introduce the first health check kiosk deployed in support centers of temporary housing communities.

\subsection{UMe-1 System}

The UMe-1 system is targeted at mobile mass medical examinations, accommodating annual health checks enforced by law: the Industrial Safety and Health Law [2] for companies and the School Health and Safety Act [3] for schools (Fig. 4). The equipment used for examinations depends on the examinee's requests and no special network configuration is needed for integrating devices because of the wireless network configuration protocol. An examination bus transports equipment and there is no wire connection among devices and the interviewer's PC. Mass medical examinations that include patient care at medical facilities are conducted in a well-structured environment with medical staff managing the procedure. Examinees can easily follow instructions 


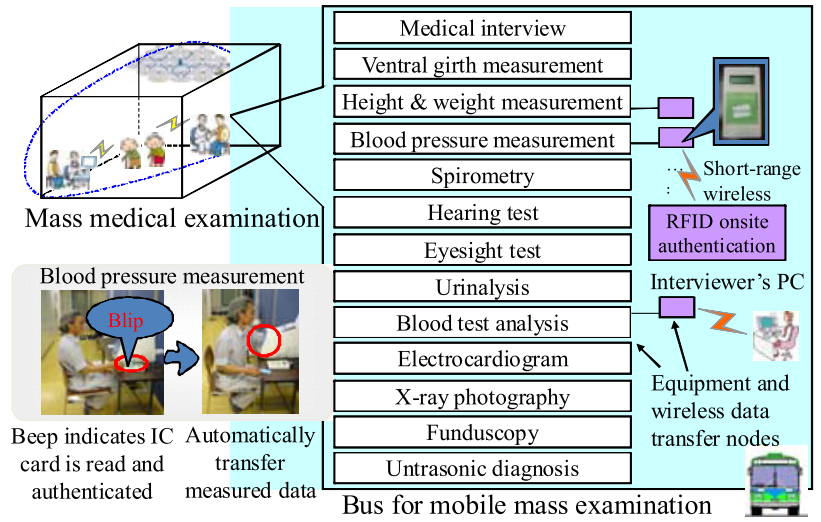

Fig. 4 UMe-1 system components and usage.

during the examination.

The UMe-1 system uses short-range wireless communication with standard crypto AES128-CBC encryption between the equipment and a data collector on the interviewer's PC. Equipment currently requires an adequate power supply and did not have a standard and efficient data protocol/format for short-range wireless communication for medical/healthcare purposes in advance of the first BAN standard IEEE802.15.6 [4] published in February 2012. In the system, we defined a protocol and data format suitable for a power-efficient wireless communication environment using battery-operated devices and satisfying the Pharmaceutical Affairs Law [5] and the protocol and data format are portable based on the IEEE802.15.6 specifications.

The system is used for self-performed health checkups. Users can check their health in a low-stress environment and save the time that would be needed for visiting a medical center. All usage cases presuppose an easy-to-use interface, IC card, and secure data storage/retrieval.

\subsection{Health Check Kiosk Version 0}

The health check kiosk version 0 is a subset of the UMe-1 system that measures blood pressure, weight, and height in a basic kiosk. Each medical instrument connects to an Android OS-based terminal (controller) via RS-232-C, and the terminal has an RF node $(2.4 \mathrm{GHz})$ and IC card reader interface. The management $\mathrm{PC}$ has the same RF interface as the coordinator to receive all data coming from the instruments and stores that data (Fig. 5).

The system adopts international standard interfaces and use open-source-based software. The wireless communication is based on the IEEE802.15.6 standard protocol and the IC card reader follows the ISO/IEC14443 standard. The coordinator receives data and securely stores them in the opensource-based database.

An examinee first receives an IC card for local user identification and independently undergoes measurements at each instrument, as shown in Fig. 5. Data are wirelessly collected in the PC at the support center. Users can at any time print out their records and consult with a doctor using
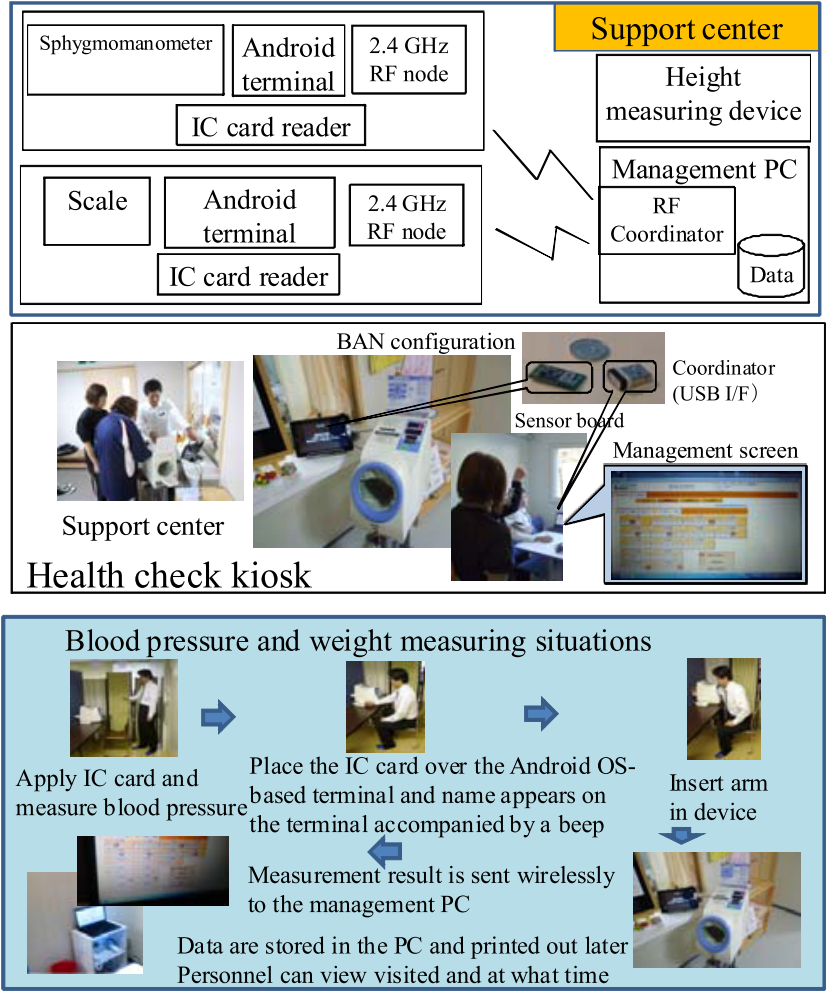

Fig.5 Health check kiosk version 0 and its usage scenario.

the records as a reference.

The important features are as follows.

1) IC cards do not store personal data

2) It is easy to reissue IC cards

3) Well-known medical instruments are used

4) Each individual's data are sent securely with no extra security operation

5) The kiosk is compact and located at the entrance of the support center

The system deploys only a sphygmomanometer because of space limitations at the center. The bloodmeasuring device is popular among all people and LSAs need not explain its utility to residents. Sophisticated but unfamiliar devices are difficult to introduce at the center because staffs are quite busy and have little time to devote to them. Sphygmomanometers are a good candidate device as a first step for the general public, which is familiar with interpreting their data.

\subsection{Next Stage: Community Health Check System}

The community health check system is the next plan for deployment in the area. The system is a community healthcare service network made up of the health check kiosk version 0 and a BAN system in each home, and connects to a backend cloud located in either a center or a virtual center consisting of small servers, such as laptop PCs, scattered across an area with distributed data management service (Fig. 6). Residents set medical instruments or wear small sensors at 


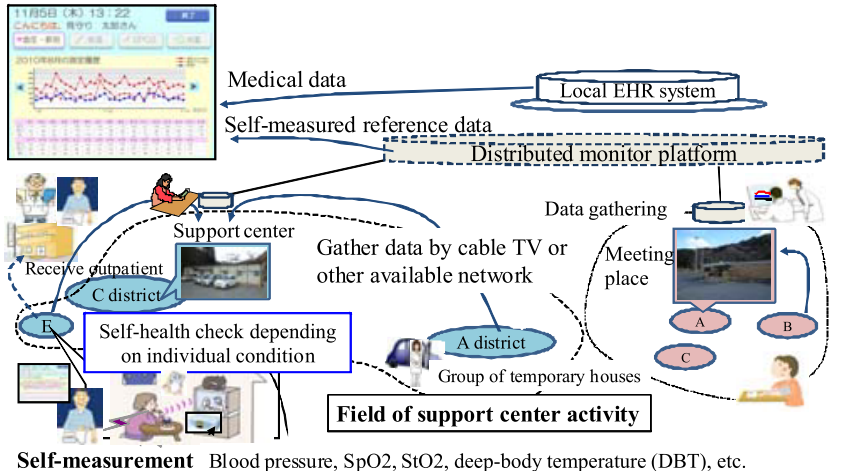

Fig. 6 Community health check system.

home depending on their health condition and preference, collect data from the devices in a coordinator at home, and upload the data to the center.

Instruments/sensors and the coordinator are configured as a BAN following the IEEE802.15.6 standard. Other radio communications, such as Bluetooth, may be used between an instrument, such as a weight scale, and the coordinator if a radio is equipped at both ends.

The most suitable network is selected based on maintainability and management cost. We will use the available cable television network to upload data to the system since all homes are equipped with cable television sets and the uplink can be used with no extra networking. Cable televisions also have an interface that is easy to use for most people. There is a possibility of deploying wireless communication from each home, but if external walls are made of metal this could decrease the radio efficacy. It is also difficult to partially reconstruct housing without the permission of the city office. A cellular service may be available, but this requires operational expense and it needs to be clear which party will bear the cost.

The system will be implemented by open source and standard web for maintainability. An open-source-code distributed file system, such as Hadoop, may be used to remove the risk of disk access failure.

\section{Body Area Network and Home}

A BAN is a short-range wireless network, consisting of sensors and a coordinator, wearable on or implantable in the human body, as shown in Fig. 7. The coordinator is a gateway enabling data exchange between outside networks and the BAN.

A BAN requires less traffic from the coordinator to sensors to reduce the receive-wait state in the sensor RF and permits plug-in of sensors, such as an $\mathrm{SpO} 2$, at setup or any time later. Sensors are light enough for users to carry them without being aware of their presence. Effective security requires simple key pre-distribution with re-keying to maintain strong security and lower-traffic mutual authentication between each sensor and the coordinator, and protects wireless communications from eavesdropping and attacks [6].

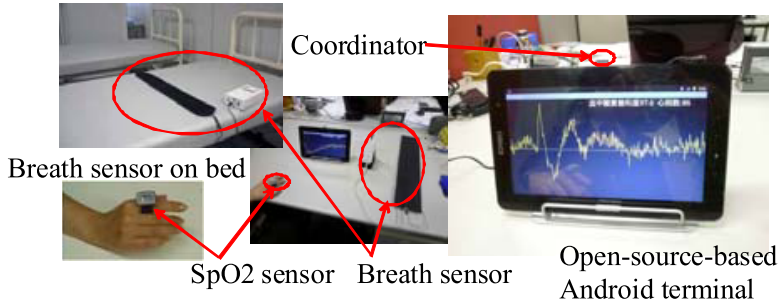

Fig. 7 Sensors and a coordinator

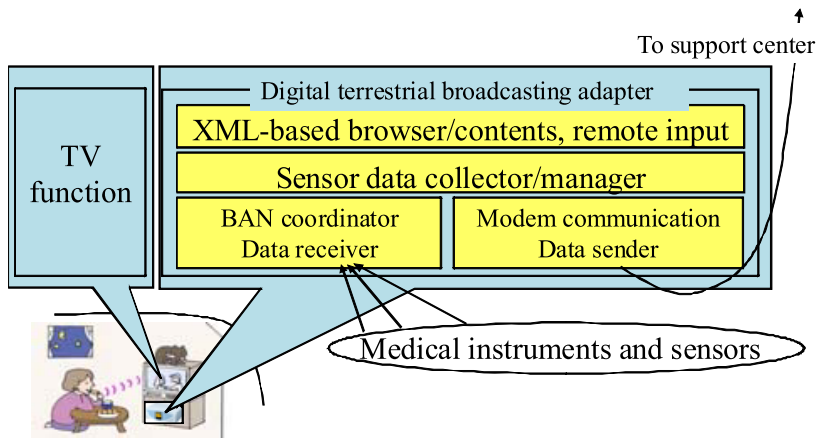

Fig. 8 Television and BAN configuration.

\subsection{Industry-Driven QoL-SN Standard Association}

The Quality-of-Life Sensing Network (QoL-SN) association [7] was established on July 5, 2010, to define industry-driven interfaces for components containing medical/healthcare-related sensors to join a BAN as per IEEE802.15.6 and for applications for analyzing medical/healthcare conditions at the request of medi$\mathrm{cal} /$ healthcare professionals and to promote them for userfriendly and cost-efficient self-care services. The health check kiosk follows the QoL-SN-defined interfaces and is implemented in support centers through the efforts of association members.

\subsection{Next Stage: In-Home BAN}

An in-home BAN consists of sensors and a coordinator. It configures any sensor, such as a sphygmomanometer, clinical thermometer, or $\mathrm{SpO} 2$ sensor, as long as it satisfies the BAN protocol and data format. Since many prefer a userfriendly interface, the television input control is used to set up the security of the BAN configuration. A digital terrestrial broadcast adapter provides Extensible Markup Language (XML)-based browsing and a BAN coordinator functions in between the television function and a broadcast station (Fig. 8). The XML-based contents can be in a backend broadcasting station if many services appear. The adapter collects sensor data and sends them via a modem to a support center. The adapter is assigned a dynamic IP address and the center has a fixed IP address in the community network. The adapter is an endpoint to the support center via the head end of the cable television company. Groups of 
temporary housing and the support center form a community network with the BAN via the cable television network. A user-friendly interface is designed by using the television interface and appears acceptable for residents.

\section{Conclusion and Future Work}

In this paper, we introduced the UMe- 1 system and its subset health check kiosk version 0 . The kiosk is user-friendly for both residents and staff and the measured data are easy to understand for not only medical/healthcare staff but also residents. It is important for staff of a support center to reduce their work burden by using such self-check kiosks as a tool for monitoring health conditions of large numbers of people in addition to face-to-face talks. Not everyone living in the targeted temporary housing currently uses the system, but the center's director understands its utility and foresees a community network to support people living in temporary housing distant from the support center. The system is technically proven, but funding is insufficient to deploy the community network. Research and development on actually deployable ICT applications is important for uncovering hidden research issues that have an impact on everyday life.

We will further investigate real requirements that people need.

\section{Acknowledgments}

We would like to thank Hiroyuki Kamata, M.D. of Morioka JRC Hospital for system suggestions and contact with the medical association and city officers and Professors Akiko Ogawa and Jun Sawamoto of Iwate Prefectural University for service suggestions and arranging meetings. We also wish to thank Takeyuki Nagato and Taiki Mori of Caunets Corporation and Eigo Fujikawa of Japan Computer Technology Corporation for their voluntary development of kiosk software.

\section{References}

[1] http://law.e-gov.go.jp/htmldata/S36/S36HO223.html (In Japanese)

[2] http://unpan1.un.org/intradoc/groups/public/documents/APCITY/ UNPAN009437.pdf

[3] http://www.japaneselawtranslation.go.jp/law/detail_main?re=01\& $\mathrm{vm}=\& \mathrm{id}=1736$ (Japanese)

[4] http://www.ieee802.org/15/pub/TG6.html

[5] http://law.e-gov.go.jp/htmldata/S35/S35HO145.html (Japanese)

[6] M. Kuroda, Y. Tamura, R. Kohno, and O. Tochikubo, "Empirical evaluation of zero-admin authentication for vital sensors in body area networks," IEEE EMBC 2008, pp.2349-2352, Aug. 2008.

[7] http://www.qolsn.org

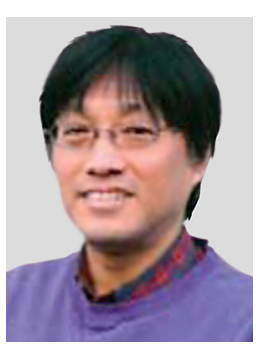

Masahiro Kuroda received the M.E. degree in systems science from the Tokyo Institute of Technology, Japan, in 1980, the M.S. degree in computer science from University of California, Santa Barbara, CA, in 1989, and received the $\mathrm{Ph} . \mathrm{D}$. degree in computer science from Shizuoka University, Japan, in 2000. He is now with NICT. 